\title{
Đa dạng hóa kinh doanh, hiệu quả và rủi ro
}

\section{The relationship between diversification strategies and risk, performance. Evidence from Vietnam's listed non-financial firms}

\author{
Vũ Hữu Thành ${ }^{1 *}$, Nguyễn Minh Hà ${ }^{1}$, Nguyễn Minh Kiều ${ }^{1}$ \\ ${ }^{1}$ Trường Đại học Mở Thành Phố Hồ Chí Minh, Việt Nam \\ "Tác giả liên hệ, Email: thanh.vh@ou.edu.vn
}

THÔNG TIN

DOI: $10.46223 / \mathrm{HCMCOUJS.}$

econ.vi.13.1.526.2018

Ngày nhận: 29/12/2017

Ngày nhận lại: 04/01/2018

Duyệt đăng: 04/01/2018

Tù khóa:

đa dạng hóa kinh doanh ngành không liên quan, đa dạng hóa kinh doanh ngành có liên quan, đa dạng hóa kinh doanh, hiệu quả, rủi ro phá sản

\section{TÓM TẮT}

Đa dạng hóa kinh doanh trong nghiên cứu này được chia làm hai loại là đa dạng hóa kinh doanh ngành có liên quan và đa dạng hóa kinh doanh ngành không liên quan. Hai loại đa dạng hóa kinh doanh vừa nêu có những đặc điểm khác nhau nên tác động của chúng tới hiệu quả và rủi ro là không đồng nhất. Mục tiêu của nghiên cứu là xem xét tác động của đa dạng hóa kinh doanh đến hiệu quả và rủi ro của các doanh nghiệp trên sàn chứng khoán Việt Nam. Đa dạng hóa kinh doanh ngành có liên quan được giả định là tác động dương tới hiệu quả và rủi ro; trong khi đó, đa dạng hóa kinh doanh ngành không liên quan được giả định ngược lại. Khi đó, mô hình lý thuyết được đề xuất là "mô hình dường như không liên quan" (SUR) và đây được coi là một dạng của mô hình cấu trúc tuyến tính (SEM). Dữ liệu nghiên cứu là những doanh nghiệp phi tài chính niêm yết trên thị trường chứng khoán Việt Nam từ 2008 tới 2015 với tổng số 3760 quan sát. Kết quả cho thấy các biến trên đã tác động đúng như kỳ vọng. Tuy nhiên, mức tác động biên tới rủi ro của biến đa dạng hóa kinh doanh ngành có liên quan và không liên quan là rất thấp. Ngoài ra, mức tác động biên tới hiệu quả của biến đa dạng hóa kinh doanh ngành có liên quan cao hơn biến đa dạng hóa kinh doanh ngành không liên quan.

\section{ABSTRACT}

Diversification strategies are classified into two types: related diversification and unrelated diversification. Related diversification is supposed to have a positive impact on risk and performance; on the other hand, unrelated diversification is supposed to affect them in reverse. Therefore, the proposed theoretical model is called a "seemingly unrelated model" (SUR), and it is considered as a form of linear structural equation model (SEM). The data was collected from non-financial firms 
Keywords:

diversification strategies, performance, related diversification, risk of bankruptcy, unrelated diversification

listed on Vietnam's stock market from 2008 to 2015 with a total of 3760 observations. The results of empirical research show that the impact of related and unrelated diversifications on risk and performance is consistent with our expectations. However, the level of the marginal effects of the diversification strategies on risk is very low. In addition, the marginal effects of the related diversification on performance are greater than those of the unrelated diversification. It implies that firms, which have an abundance of resources and priority in enhancing performance, may implement a strategy of diversifying businesses into related industries.

\section{Giới thiệu}

Đa dạng hóa được coi là một trong những hoạt động mang tính chiến lược của doanh nghiệp, nó đại diện cho những quyết định quản trị chiến lược quan trọng nhất và có liên hệ mật thiết tới các kết quả đầu ra của doanh nghiệp (Ramanujam \& Varadarajan, 1989). Knecht (2013) cũng khẳng định rằng đa dạng hóa có những ảnh hưởng to lớn tới hiệu quả của doanh nghiệp. Tuy nhiên, đa dạng hóa vốn là con dao hai lưỡi: có thể phá hủy hoặc gia tăng hiệu quả và nhiều nghiên cứu thực nghiệm đã chứng minh nhận định trên (Palich, Cardinal, \& Miller, 2000).

Bên cạnh hiệu quả, rủi ro cũng là một biến được đặc biệt quan tâm trong quản trị chiến lược của doanh nghiệp. Mối liên hệ giữa đa dạng hóa và rủi ro đã sớm được quan tâm bởi Bettis và Hall (1982) và từ đó hình thành nên một hướng nghiên cứu cho tới ngày nay. Tác động của đa dạng hóa tới rủi ro cũng khá phức tạp. Tính phức tạp này là do sự đa dạng về loại hình đa dạng hóa của doanh nghiệp, từng loại hình đa dạng hóa lại được phân chia thành các loại hình đa dạng hóa cấp thấp hơn căn cứ vào các đặc điểm phân chia khác nhau. Các loại hình đa dạng hóa ở cấp thấp hơn lại có chiều hướng tác động khác biệt nhau tới rủi ro và chính bản thân từng loại hình đa dạng hóa này có thể có những mối liên hệ nhất định với nhau. Ví dụ, trong doanh nghiệp, đa dạng hóa kinh doanh được chia thành hai loại hình đa dạng có đặc điểm khác biệt nhau là đa dạng hóa kinh doanh ngành có liên quan và đa dạng hóa kinh doanh ngành không liên quan tới ngành kinh doanh chính. Đa dạng hóa kinh doanh ngành có liên quan có thể làm gia tăng hiệu quả (Palich et al., 2000) nhưng đồng thời cũng làm gia tăng rủi ro đặc thù (Bettis \& Hall, 1982). Trong khi đó, đa dạng hóa lĩnh vực không liên quan có tác động ngược lại.

Nghiên cứu này thay vì đi tìm hiểu tác động riêng rẽ của từng loại hình đa dạng hóa tới hiệu quả và rủi ro thì sẽ tìm hiểu tác động đồng thời của đa dạng hóa kinh doanh ngành có liên quan và đa dạng hóa kinh doanh ngành không liên quan tới hiệu quả và rủi ro trong cùng một mô hình cấu trúc tuyến tính SEM. 


\section{Cơ sở lý thuyết}

\section{1. Đa dạng hóa và hiệu}

\section{Đa dạng hóa}

Kenny (2009) đã nhận định rằng việc trả lời cho câu hỏi thế nào là đa dạng hóa là không dễ dàng đối với cả các nhà nghiên cứu và nhà quản lý. Tính phức tạp của nó còn được thể hiện bởi nhận định của Hall và John (1994), Lang và Stulz (1993), Lubatkin, Merchant, và Srinivasan (1993), hay Robins và Wiersema (2003) khi các tác giả cho rằng đa dạng hóa được coi là một thang đo đa hướng, nó được cấu thành bởi rất nhiều các thang đo thành phần. Đa dạng hóa của Rumelt (1974) được sử dụng rộng rãi trong nhiều nghiên cứu thực nghiệm sau này vì quan điểm của tác giả là không quan tâm tới việc doanh nghiệp thay đổi bên trong nội bộ như thế nào khi tiến hành đa dạng hóa. Rumelt (1974) chỉ căn cứ vào đặc tính của sản phẩm đầu ra và tỷ trọng doanh thu của những loại hình sản phẩm này so với tổng số để xác định đa dạng hóa. Nghiên cứu sẽ tiếp cận đa dạng hóa kinh doanh theo phân loại của Rumelt (1974) nhưng chia thành hai loại chính là đa dạng hóa lĩnh vực có liên quan và đa dạng hóa lĩnh vực không liên quan. Hai loại hình đa dạng hóa này được sử dụng phổ biến trong các nghiên cứu thực nghiệm về mối liên hệ giữa đa dạng hóa kinh doanh, hiệu quả và rủi ro.

Đa dạng hóa kinh doanh ngành có liên quan: Doanh nghiệp kinh doanh những sản phẩm khác ngành cấp 3 nhưng cùng ngành cấp 2 của ngành kinh doanh chính (Rumelt, 1982).

Đa dạng hóa kinh doanh ngành không liên quan: Doanh nghiệp kinh doanh những sản phẩm khác ngành cấp hai của ngành kinh doanh chính (Rumelt, 1982).

\section{Hiệu quả của doanh nghiệp}

Hiệu quả của doanh nghiệp có thể được hiểu như là kết quả đầu ra của các hoạt động trong doanh nghiệp và kết quả này cần định lượng được (Neely, Gregory, \& Platts, 1995). Trong doanh nghiệp, có rất nhiều các hoạt động khác nhau vì thế có rất nhiều góc nhìn khác nhau về hiệu quả và vấn đề đặt ra ở đây là "hiệu quả" dùng để làm gì? (Santos \& Brito, 2012). Hitt (1988) đưa ra ý kiến rằng "hiệu quả” phải luôn được đo lường và kết quả đo lường này là để phục vụ cho các bên liên quan (stakeholders) như cổ đông, nhà quản lý, xã hội, công nhân... Với mỗi bên liên quan khác nhau thì các nhìn nhận về hiệu quả dưới góc độ đo lường là khác nhau. Nghiên cứu sẽ tiếp cận khái niệm hiệu quả của doanh nghiệp dưới góc độ của cổ đông (một bên liên quan của doanh nghiệp) và tương ứng với góc nhìn này là hiệu quả về tài chính của doanh nghiệp.

\section{Đa dạng hóa và hiệu quả}

Lý thuyết về tính kinh tế theo phạm vi (economies of scope) đề cập tới sự thay đổi chi phí sản xuất trung bình khi doanh nghiệp mở rộng sản xuất sản phẩm. Khi doanh nghiệp thực hiện tính kinh tế theo quy mô hay nói cách khác doanh nghiệp đa dạng hóa những sản phẩm mà việc đa dạng hóa này có sử dụng những nguồn lực dùng chung và giúp cho chi phí kết hợp giảm xuống. Kết quả là lợi nhuận của doanh nghiệp sẽ tăng lên. Thông thường, tính phi kinh tế theo phạm vi diễn ra khi doanh nghiệp thực hiện đa dạng hóa theo chiều dọc hay đa dạng hóa ở ngành kinh doanh không liên quan (Pils, 2009). Ngoài ra nếu doanh nghiệp thực hiện đa dạng 
hóa quá mức hay kinh doanh ở nhiều lĩnh vực khác nhau (dù có liên quan hay không liên quan tới lĩnh vực kinh doanh chính) sẽ khiến cho việc chia sẻ những nguồn lực dùng chung trở nên khó khăn và tính hiệu quả sẽ giảm (Palich et al., 2000). Lý thuyết này được dùng để lý giải mối liên hệ tích cực giữa đa dạng hóa kinh doanh ngành có liên quan tới hiệu quả kinh doanh. Khi doanh nghiệp đa dạng hóa kinh doanh có ngành liên quan, doanh nghiệp sẽ có cơ hội sử dụng các nguồn lực dùng chung và từ đó tiết giảm được chi phí. Kết quả là hiệu quả kinh doanh của doanh nghiệp có thể tăng lên.

Wernerfelt (1984) và Barney (1991) phát triển lý thuyết về quan điểm phát triển dựa vào nguồn lực (theory of resource-based view - RBV) từ lý thuyết tăng trưởng doanh nghiệp của Penrose (1959). Lý thuyết này nhận định khi các nguồn lực của doanh nghiệp trở nên dư thừa và dòng tiền tự do ngày càng lớn, doanh nghiệp sẽ tiến hành đa dạng hóa. Lúc đó, những tài sản đặc thù nằm trong nguồn lực sẵn có của doanh nghiệp tuy tạo ra lợi thế cạnh tranh bền vững nhưng chính nó lại là trở ngại trong việc chuyển đổi nguồn lực sang khía cạnh kinh doanh mới. Do vậy giá trị mà đa dạng hóa đem lại sẽ phụ thuộc vào sự phù hợp giữa một bên là nguồn lực sẵn có của doanh nghiệp và một bên là lĩnh vực kinh doanh mà doanh nghiệp sẽ thâm nhập. Lý luận này gợi ý rằng doanh nghiệp nên đa dạng hóa vào những ngành có liên quan để sự chuyển đổi nguồn lực diễn ra dễ dàng. Ngược lại, việc đa dạng hóa vào những ngành không liên quan sẽ khiến cho việc chuyển đổi trở nên khó khăn hơn và kéo theo hiệu quả sẽ giảm (Wan, Hoskisson, Short, \& Yiu, 2010).

Căn cứ vào các nghiên cứu lý thuyết và thực nghiệm, Nghiên cứu đưa ra giả thuyết về mối liên hệ giữa đa dạng hóa kinh doanh và hiệu quả như sau:

H1: Đa dạng hóa kinh doanh ngành có liên quan có xu hướng tác động duơng đối với hiệu quả kinh doanh của doanh nghiệp

H2: Đa dạng hóa kinh doanh ngành không liên quan có xu huớng tác động âm tới hiệu quả kinh doanh của doanh nghiệp

\section{2. Đa dạng hóa kinh doanh và rủi ro}

\section{Rủi ro}

Có rất nhiều các loại rủi ro khác nhau trong doanh nghiệp trong đó rủi ro phá sản là loại rủi ro được quan tâm nhiều nhất vì nó ảnh hưởng tới nhiều bên liên quan như cổ đông, đối tác, hay người cho vay... Nghiên cứu này sẽ tiếp cận rủi ro theo hướng rủi ro phá sản của doanh nghiệp. Phá sản thường bắt đầu từ tình huống khó khăn về tài chính và sau đó là tới sự can thiệp của luật pháp nếu sự khó khăn đó khiến cho doanh nghiệp không có khả năng trả được nợ (Karels \& Prakash, 1987).

Việc đo lường rủi ro của doanh nghiệp phụ thuộc vào phân loại rủi ro, có nghĩa là rủi ro được nhìn ở góc độ nào thì sẽ có cách tính toán tương ứng. Theo Peirson, Brown, Easton, và Howard (2014), rủi ro của doanh nghiệp, về mặt tổng quát, có thể được phân làm hai loại: (i) rủi ro hệ thống (systematic risk) và (ii) rủi ro phi hệ thống (unsystematic risk). Nghiên cứu này sẽ tiếp cận đo lường rủi ro theo hướng rủi ro phi hệ thống và cụ thể hơn đó là rủi ro phá sản được đo lường thông qua các chỉ số tài chính, một loại rủi ro đặc thù của doanh nghiệp. Thêm vào đó, rủi ro phá sản vốn được quan tâm phổ biến và sẵn có nhiều mô hình đo lường khác 
nhau. Nghiên cứu này lựa chọn mô hình đo lường Merton - KMV để tính toán xác suất phá sản trong một khoảng thời gian cụ thể thông qua việc tính toán "khoảng cách tới thời điểm phá sản”. Mô hình đã được Merton (1974) xây dựng và được ba tác giả Kealhofer (Kealhofer \& Bohn, 1998), McQuown (McQuown, 1997) và Vasicek (Vasicek, 1984) phát triển.

\section{Đa dạng hóa và rủi ro}

Sự tác động của đa dạng hóa tới rủi ro phá sản khá phức tạp vì đa dạng hóa được phân thành hai loại là đa dạng hóa có liên quan và đa dạng hóa không liên quan. Nghiên cứu sẽ lần lượt tìm hiểu góc nhìn của Lubatkin và Chatterjee (1994) và Bettis và Hall (1982) về rủi ro của đa dạng hóa.

\section{(i) Nghiên cứu của Lubatkin và Chatterjee (1994)}

Lubatkin và Chatterjee (1994) đã kết luận rằng đa dạng hóa kinh doanh ngành có liên quan sẽ làm giảm rủi ro phi hệ thống; ngược lại, đa dạng hóa ngành không liên quan sẽ làm tăng rủi ro phi hệ thống. Nghiên cứu giải thích rằng khi thực hiện đa dạng hóa có liên quan, doanh nghiệp vốn sở hữu những nguồn lực có mối liên hệ tương hỗ (synergistic interrelationship). Những mối liên hệ này bao gồm mối liên hệ hữu hình và vô hình đã bổ sung cho nhau trong quá trình mở rộng kinh doanh và giúp cho doanh nghiệp giảm thiểu rủi ro đặc thù. Ngược lại, khi doanh nghiệp mở rộng hoạt động kinh doanh sang những ngành không liên quan thì các nguồn lực được sử dụng để đầu tư rất khó có mối liên hệ hỗ trợ lẫn nhau và vì vậy sẽ làm gia tăng rủi ro phi hệ thống.

\section{(ii) Lý thuyết của Bettis và Hall (1982) về rủi ro đa dạng hóa}

Nghiên cứu lý thuyết của Bettis và Hall (1982) về tác động của đa dạng hóa kinh doanh và rủi ro đã phân chia tác động của hai loại hình đa dạng hóa là đa dạng hóa có liên quan và đa dạng hóa không liên quan tới rủi ro đặc thù và mỗi loại hình đa dạng hóa có hình thức tác động khác nhau. Khi doanh nghiệp cùng kinh doanh ở hai lĩnh vực có liên quan tới nhau thì lợi nhuận có xu hướng di chuyển cùng chiều với nhau và tạo ra hệ số tương quan dương. Nếu doanh nghiệp kinh doanh ở hai ngành không liên quan thì do ảnh hưởng của yếu tố chu kỳ kinh doanh hoặc đặc điểm ngành mà lợi nhuận ở hai ngành sẽ có xu hướng di chuyển ngược chiều nhau và hệ số tương quan sẽ âm. Do vậy Bettis và Hall (1982) đã đưa ra giả thuyết rằng doanh nghiệp đa dạng hóa kinh doanh ở lĩnh vực có liên quan sẽ làm tăng rủi ro trong khi đó doanh nghiệp đa dạng hóa kinh doanh ở lĩnh vực không liên quan lại làm giảm rủi ro đặc thù của doanh nghiệp.

Nghiên cứu của Lubatkin và Chatterjee (1994) và Bettis và Hall (1982) có cách tiếp cận khác nhau và đưa ra kết luận trái ngược nhau. Lubatkin và Chatterjee (1994) nhận định rằng đa dạng hóa sang ngành kinh doanh có liên quan làm giảm rủi ro đặc thù và đa dạng dạng hóa sang ngành kinh doanh không liên quan làm tăng rủi ro đặc thù. Bettis và Hall (1982) đưa ra cách nhìn ngược lại. Nghiên cứu nhận thấy kết luận của Bettis và Hall (1982) phù hợp với hoạt động đa dạng hóa hướng ra môi trường kinh doanh bên ngoài của doanh nghiệp. Ngược lại, góc nhìn của Lubatkin và Chatterjee (1994) phù hợp với hoạt động đa dạng hóa nguồn lực hướng vào môi trường bên trong. Nghiên cứu nghiêng về quan điểm của Bettis và Hall (1982) vì biến được nghiên cứu liên quan tới môi trường kinh doanh bên ngoài của doanh nghiệp. 
Từ khảo cứu lý thuyết và nghiên cứu thực nghiệm trước, nghiên cứu đề xuất giả thuyết về mối liên hệ giữa đa dạng hóa kinh doanh và rủi ro phá sản như sau:

H3: Rủi ro phá sản của doanh nghiệp có thể sẽ gia tăng khi doanh nghiệp thục hiện đa dạng hóa kinh doanh ngành có liên quan

H4: Rủi ro phá sản của doanh nghiệp có thể sẽ giảm nếu doanh nghiệp tiến hành đa dạng hóa kinh doanh ngành không liên quan

Căn cứ vào giả thuyết $\mathrm{H} 1, \mathrm{H} 2, \mathrm{H} 3$, và $\mathrm{H} 4$, nghiên cứu đề xuất mô hình mối liên hệ giữa các yếu tố đa dạng hóa kinh doanh, hiệu quả và rủi ro như sau:

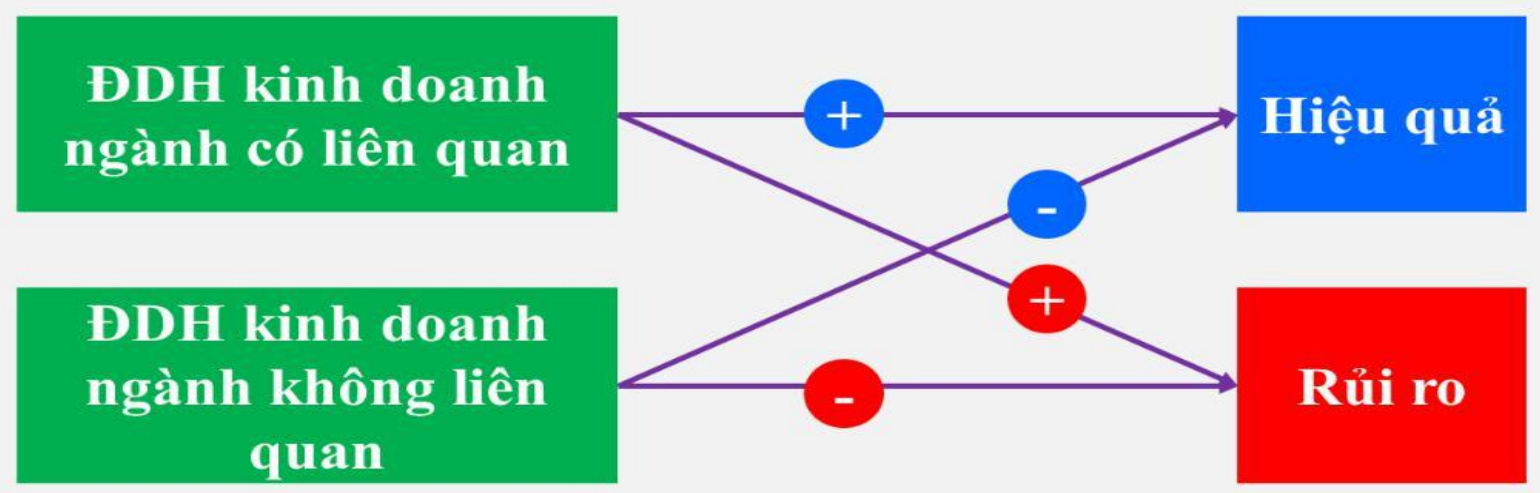

Hình 1. Mối liên hệ giữa đa dạng hóa kinh doanh với hiệu quả và rủi ro

\section{Phương pháp nghiên cứu}

\subsection{Mô hình wớc lự̛ng}

Từ mô hình nghiên cứu, nghiên cứu đề xuất mô hình ước lượng thực nghiệm, bao gồm một hệ hai phương trình như sau:

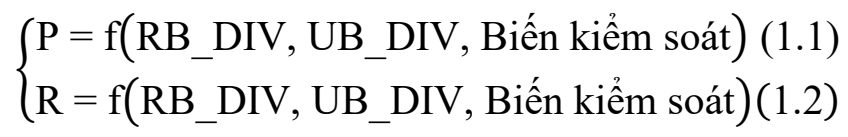

Trong đó: $\mathrm{P}$ là hiệu quả hoạt động của doanh nghiệp, $\mathrm{R}$ là rủi ro, RB_DIV thể hiện mức độ đa dạng hóa kinh doanh ở lĩnh vực có liên quan, UB_DIV đại diện cho mức độ đa dạng hóa ở lĩnh vực không liên quan.

\section{2. Đo lường các biến}

\section{(i) Biến hiệu quả}

Nghiên cứu tiếp cận khái niệm "hiệu quả doanh nghiệp" từ góc nhìn của cổ đông nên tương ứng với góc nhìn này sẽ là khía cạnh lợi nhuận của doanh nghiệp. Đây cũng là thang đo hiệu quả về mặt kế toán của doanh nghiệp và được sử dụng phổ biến bởi nhiều nghiên cứu (Lee $\& \mathrm{Li}, 2012)$. Chỉ số được dùng để đo lường hiệu quả ở góc độ cổ đông là "lợi nhuận sau thuế chia vốn chủ sở hữu" - ROE (Return on Equity). 


\section{(ii) Biến rủi ro}

Căn cứ vào nghiên cứu của Kealhofer và Bohn (1998), McQuown (1997) và Vasicek (1984), nghiên cứu sẽ tính toán khoảng cách tới phá sản và từ đó ước lượng được xác suất phá sản của doanh nghiệp trong thời hạn một năm như sau:

$$
D D=\frac{\operatorname{Ln} \frac{V}{D_{p t}}+\left(r-\frac{\sigma_{V}^{2}}{2}\right) t}{\sigma_{V} \sqrt{t}}
$$

Trong đó: $\mathrm{DD}$ là khoảng cách tính tới thời điểm phá sản. $\mathrm{V}$ và $\sigma_{\mathrm{E}}$ được tính toán từ mô hình định giá quyền chọn Black-Scholes cho giá trị vốn cổ phần $\left(E=V \Omega\left(\mathrm{d}_{1, \mathrm{t}}\right)-\mathrm{e}^{\mathrm{r}(\mathrm{T}-\mathrm{t})} \mathrm{B} . \Omega\left(\mathrm{d}_{2, \mathrm{t}}\right)\right)$. $\mathrm{r}$ là lãi suất phi rủi ro. $\sigma^{2} \mathrm{v}$ là phương sai của $\mathrm{V}$. $\mathrm{D}_{\mathrm{pt}}$ là điểm phá sản. $\mathrm{t}$ được chọn là 1 năm.

Khi đó xác suất phá sản trong vòng 1 năm - EDF được tính như sau:

$$
E D F=\operatorname{Pr}=N(-D D)
$$

EDF là xác suất cộng dồn của hàm phân phối chuẩn hóa tại điểm -DD. Nếu DD càng lớn thì EDF càng nhỏ và ngược lại.

\section{(iii) Biến đa dạng hóa kinh doanh}

Đa dạng hóa kinh doanh bao gồm hai biến: đa dạng hóa kinh doanh ngành có liên quan (RB_DIV) và đa dạng hóa kinh doanh ngành không liên quan (UB_DIV). Cả hai biến số đều có đơn vị là bit. Nghiên cứu này sẽ kết hợp phương pháp phân loại của Rumelt (1974) và phương pháp tính toán entropy.

\section{Múc độ đa dạng hóa kinh doanh ngành có liên quan:}

$$
R B_{-} D I V=\sum_{r=1}^{n} p_{r} \log _{2}\left(\frac{1}{p_{r}}\right)
$$

Trong đó, $\mathrm{r}=$ lĩnh vực kinh doanh có liên quan tới lĩnh vực kinh doanh chính, $\mathrm{p}_{\mathrm{r}}$ là tỷ lệ của doanh thu từng ngành kinh doanh có liên quan trên tổng doanh thu.

Múc độ đa dạng hóa kinh doanh ngành không liên quan:

$$
U B \_D I V=\sum_{u=1}^{n} p_{u} \log _{2}\left(\frac{1}{p_{u}}\right)
$$

Trong đó, $\mathrm{u}=$ lĩnh vực kinh doanh không liên quan tới lĩnh vực kinh doanh chính, pu là tỷ lệ của từng ngành kinh doanh không liên quan trên tổng doanh thu.

\section{(iv) Các biến kiểm soát}

Các biến kiểm soát trong mô hình được chia làm hai nhóm, nhóm biến kiểm soát tác động tới hiệu quả và nhóm biến tác động tới rủi ro. Thông qua khảo sát nghiên cứu trước, nghiên cứu đề xuất hai nhóm biến kiểm soát được mô tả ở hai bảng như sau: 


\section{Bảng 1}

Tổng hợp các biến số kiểm soát tác động tới hiệu quả kinh doanh

\begin{tabular}{|c|c|c|c|c|}
\hline Ký hiệu & Tên gọi & Cách tính & Kỳ vọng & Tác giả \\
\hline LNA & Quy mô công ty & Ln tổng tài sản & - & Shepherd (1986) \\
\hline SGA_S & $\begin{array}{l}\text { Tỷ lệ chi phí hoạt } \\
\text { động trên doanh thu }\end{array}$ & $\begin{array}{l}\text { Chi phí hoạt } \\
\text { động/Doanh thu thuần }\end{array}$ & - & $\begin{array}{l}\text { Kaplan và Cooper } \\
\text { (1998) }\end{array}$ \\
\hline SG & $\begin{array}{l}\text { Tăng trưởng doanh } \\
\text { thu }\end{array}$ & $\begin{array}{l}\text { (Doanh thu } \mathrm{t}_{\mathrm{t}}-\text { Doanh } \\
\left.\text { thu } \mathrm{t}_{\mathrm{t}-1}\right) / \text { Doanh thu } \mathrm{t}-1\end{array}$ & + & $\begin{array}{l}\text { Brush, Bromiley, } \\
\text { và Hendrickx } \\
(2000)\end{array}$ \\
\hline AGE & $\begin{array}{l}\text { Tuồi đời doanh } \\
\text { nghiệp }\end{array}$ & $\begin{array}{l}\text { (Năm đánh giá - năm } \\
\text { đăng ký kinh doanh) }+1\end{array}$ & + & Glancey (1998) \\
\hline CR & $\begin{array}{l}\text { Tỷ lệ thanh toán hiện } \\
\text { hành }\end{array}$ & $\begin{array}{l}\text { Nợ ngắn hạn/Tài sản } \\
\text { ngắn hạn }\end{array}$ & - & Appuhami (2008) \\
\hline $\mathrm{CCC}$ & $\begin{array}{l}\text { Chu kỳ luân chuyển } \\
\text { tiền }\end{array}$ & $\begin{array}{l}\text { Số ngày tồn kho + Số } \\
\text { ngày phải thu - Số ngày } \\
\text { phải trả }\end{array}$ & - & Deloof (2003) \\
\hline $\mathbf{A S}$ & Cấu trúc tài sản & $\begin{array}{l}\text { Tài sản dài hạn/Tài sản } \\
\text { ngắn hạn }\end{array}$ & + & $\begin{array}{l}\text { Afza và Nazir } \\
(2007)\end{array}$ \\
\hline $\mathbf{A T}$ & $\begin{array}{l}\text { Vòng quay tổng tài } \\
\text { sản }\end{array}$ & $\begin{array}{l}\text { Doanh thu thuần/Tổng } \\
\text { tài sản }\end{array}$ & + & $\begin{array}{l}\text { Jansen, Ramnath, } \\
\text { và Yohn (2012) }\end{array}$ \\
\hline
\end{tabular}

Nguồn: Kết quả phân tích dữ liệu của nhóm nghiên cứu

\section{Bảng 2}

Tổng hợp các biến số kiểm soát tác động tới rủi ro

\begin{tabular}{|l|l|l|c|l|}
\hline \multicolumn{1}{|c|}{ Ký hiệu } & \multicolumn{1}{|c|}{ Tên gọi } & \multicolumn{1}{c|}{ Cách tính } & Kỳ vọng & \multicolumn{1}{c|}{ Tác giả } \\
\hline LNA & Logarit tổng tài sản & Ln tổng tài sản & - & Dichev (1998) \\
\hline SGA_S & $\begin{array}{l}\text { Tỷ lệ chi phí hoạt } \\
\text { dộng trên doanh thu }\end{array}$ & $\begin{array}{l}\text { Chi phí hoạt } \\
\text { dộng/Doanh thu thuần }\end{array}$ & + & $\begin{array}{l}\text { Kaplan và Cooper } \\
\text { (1998) }\end{array}$ \\
\hline CR & $\begin{array}{l}\text { Tỷ số thanh toán } \\
\text { hiện hành }\end{array}$ & $\begin{array}{l}\text { Nợ ngắn hạn/Tài sản } \\
\text { ngắn hạn }\end{array}$ & - & $\begin{array}{l}\text { Dambolena và } \\
\text { Khoury (1980) }\end{array}$ \\
\hline CCC & $\begin{array}{l}\text { Chu kỳ luân chuyển } \\
\text { tiền }\end{array}$ & $\begin{array}{l}\text { Số ngày tồn kho + Số } \\
\text { ngày phải thu - Số ngày } \\
\text { phải trả }\end{array}$ & - & Deloof (2003) và \\
\hline AT & $\begin{array}{l}\text { Vòng quay tổng tài } \\
\text { sản }\end{array}$ & $\begin{array}{l}\text { Doanh thu thuần/Tồng } \\
\text { tài sản }\end{array}$ & - & $\begin{array}{l}\text { Pan và David } \\
\text { (2000) }\end{array}$ \\
\hline P_B & $\begin{array}{l}\text { Tỷ lệ vốn hóa thị } \\
\text { trường chia vốn chủ } \\
\text { sở hữu }\end{array}$ & $\begin{array}{l}\text { Giá trị thị trường của cổ } \\
\text { phiếu/Giá trị sổ sách của } \\
\text { cồ phiếu }\end{array}$ & - & Dempsey (2010) \\
\hline OCF_S & $\begin{array}{l}\text { Tỷ lệ dòng tiền hoạt } \\
\text { động chia doanh thu }\end{array}$ & $\begin{array}{l}\text { Dòng tiền hoạt } \\
\text { động/Doanh thu }\end{array}$ & - & $\begin{array}{l}\text { Giacomino và } \\
\text { Mielke (1993) }\end{array}$ \\
\hline EBIT_I & $\begin{array}{l}\text { Khả năng thanh toán } \\
\text { lãi vay }\end{array}$ & EBIT/I & $\begin{array}{l}\text { Claessens, } \\
\text { Djankov, và } \\
\text { Nenova (2001) }\end{array}$ \\
\hline
\end{tabular}

Nguồn: Kết quả phân tích dữ liệu của nhóm nghiên cứu 


\subsection{Phương pháp ước lự̣ng}

Mô hình cấu trúc (Structural Equation Model - SEM) còn gọi là mô hình hồi quy đồng thời (Simultaneous Equation Model - SEM) (Fox, 2006). Mô hình nghiên cứu có hai biến phụ thuộc là ROE và RISK. Hai biến phụ thuộc này lại chịu tác động chung của các biến như RB_DIV, UR_DIV, LNA, SGA_S, CCC, CR và AT. Ngoài ra, hai biến phụ thuộc trên còn bị tác động riêng rẽ bởi một số biến khác như ROE bị tác động bởi AGE và AS; RISK bị tác động riêng rẽ bởi $\mathrm{P} \_\mathrm{B}, \mathrm{OCF} S \mathrm{~S}, \mathrm{EBIT} I$. Một cách tổng quát, mô hình nghiên cứu có dạng "mô hình dường như không liên quan" (SUR - Seemingly unrelated model) và SUR cũng được coi là một dạng của mô hình SEM (Stata, 2015).

Hệ phương trình của mô hình SEM (còn gọi là mô hình hồi quy đồng thời) của nghiên cứu được mô tả chi tiết như sau:

$$
\left\{\begin{array}{c}
\mathrm{P}=\mathrm{f}(\mathrm{RB} \text { _DIV, UB_DIV, LNA, SGA_S, CCC, CR, AT, AGE và AS })(1.1) \\
\mathrm{R}=\mathrm{f}(\mathrm{RB} \text { _DIV, UB_DIV, LNA, SGA_S, CCC, CR, AT, P_B, OCF_S, EBIT_I })(1.2)
\end{array}\right.
$$

\subsection{Dũ liệu nghiên cứu}

Dữ liệu là những doanh nghiệp phi tài chính niêm yết trên thị trường chứng khoán Việt Nam thời điểm 2008 tới 2015. Các doanh nghiệp cần thỏa mãn một số điều kiện như sau: (i) Có thời gian hoạt động trên thị trường chứng khoán từ 2008 tới 2015; (ii) Được phân ngành theo GICS hoặc có thể quy đổi ngành kinh doanh tương đương theo GICS; (iii) Không gặp tình huống kinh doanh bất thường (không xuất hiện doanh thu, hủy niêm yết sau đó niêm yết lại...); (iv) Các doanh nghiệp không được phân ngành kinh doanh chính trong lĩnh vực tài chính. Cuối cùng, 470 doanh nghiệp được lựa chọn (tổng hai sàn giao dịch có khoảng 700 doanh nghiệp được niêm yết), với tổng số quan sát là 3760 (=470 x 8).

\section{Phân tích và thảo luận kết quả}

\subsection{Thống kê mô tả các biến trong mô hình}

Mô hình nghiên cứu sử dụng hai biến đại diện làm biến phụ thuộc là "Lợi nhuận trên vốn chủ sở hữu” (ROE) và "Rủi ro phá sản” (Risk). Biến ROE có giá trị nhỏ nhất đạt 0.0016 và lớn nhất đạt 20.18 . Xét ở giá trị trung bình, biến này đạt 0.147 . Như vậy xét ở khía cạnh tổng thể trong cả thời kỳ từ 2008 tới 2015, một doanh nghiệp trong mẫu nghiên cứu có tỷ suất lợi nhuận trên vốn chủ sở hữu đạt $14.7 \%$. Rủi ro phá sản, hay rủi ro tính tới ngày phá sản tính trung bình trong thời kỳ của một doanh nghiệp là $18.6 \%$ (nhỏ nhất đạt $0.2 \%$ và lớn nhất đạt $72 \%$ ). Có nghĩa, xác suất phá sản trong vòng một năm tới của một doanh nghiệp đạt trung bình là $18.6 \%$.

Biến đa dạng hóa kinh doanh được coi là biến độc lập chính trong mô hình, mức độ đa dạng hóa doanh thu ngành có liên quan có giá trị trung bình là 0.76 (bit), giá trị lớn nhất đạt 1.15 (bit) và nhỏ nhất là bằng 0 . Trong khi đó, mức độ đa dạng hóa doanh thu ngành không liên quan có giá trị trung bình là 0.45 (bit), giá trị lớn nhất đạt 1.01 (bit) và nhỏ nhất là bằng 0 .

Các biến kiểm soát khác xuất hiện trong mô hình cũng được thống kê tại Bảng sau: 


\section{Bảng 3}

Thống kê mô tả các biến kiểm soát

\begin{tabular}{|c|c|c|c|c|}
\hline Biến số & Nhỏ nhất & Lớn nhất & Trung bình & Lệch chuẩn \\
\hline LNA & 12.70 & 31.91 & 26.44 & 1.47 \\
\hline AS & 0.01 & 1.12 & 0.39 & 0.22 \\
\hline CR & 0.05 & 45.10 & 1.45 & 1.34 \\
\hline AT & 0.05 & 10.96 & 1.22 & 1.11 \\
\hline CCC & -117.80 & 1516.41 & 177.10 & 134.30 \\
\hline OCF_S & 0.001 & 0.95 & 0.14 & 1.20 \\
\hline SG & -0.98 & 67.52 & 0.13 & 1.55 \\
\hline SGA_S & 0.03 & 23.14 & 0.14 & 0.71 \\
\hline P_B & 0.70 & 24.58 & 1.05 & 1.14 \\
\hline EBIT_I & 0.31 & 7752.11 & 961.34 & 7197.40 \\
\hline AGE & 2.00 & 32.00 & 17.04 & 9.03 \\
\hline
\end{tabular}

Nguồn: Kết quả xử lý từ dữ liệu điều tra

\subsection{Kết quả hồi quy}

Nghiên cứu thực hiện hồi quy SEM và kết quả được tóm tắt như sau:

\section{Bảng 4}

Kết quả hồi quy mô hình SEM

\begin{tabular}{|c|c|c|c|c|}
\hline & Coef. & Std. Err. & $\mathbf{z}$ & $P>Z$ \\
\hline \multicolumn{5}{|l|}{$\rightarrow$ ROE } \\
\hline AGE & -0.00089 & 0.0009 & -0.97 & 0.332 \\
\hline SG & 0.00013 & 0.0004 & 0.3 & 0.760 \\
\hline CR & 0.00008 & 0.0019 & 0.04 & 0.968 \\
\hline SGA_S & $-0.03287(* * *)$ & 0.0120 & -2.74 & 0.006 \\
\hline LNA & $0.00465(* * *)$ & 0.0007 & 6.36 & 0.000 \\
\hline $\mathrm{CCC}$ & 0.00000 & 0.0000 & -0.55 & 0.583 \\
\hline AS & $-0.16060(* * *)$ & 0.0261 & -6.16 & 0.000 \\
\hline AT & $0.04181(* * *)$ & 0.0077 & 5.45 & 0.000 \\
\hline RB_DIV & $0.09573(* * *)$ & 0.0028 & 34.68 & 0.000 \\
\hline UB_DIV & $-0.12298(* * *)$ & 0.0012 & -99.32 & 0.000 \\
\hline _cons & $0.07043(* *)$ & 0.0315 & 2.23 & 0.026 \\
\hline \multicolumn{5}{|l|}{$\rightarrow$ RISK } \\
\hline $\mathrm{CR}$ & -0.00100 & 0.0013 & -0.75 & 0.456 \\
\hline SGA_S & $0.02755(* * *)$ & 0.0084 & 3.28 & 0.001 \\
\hline EBIT_I & $0.00000(* *)$ & 0.0000 & 1.19 & 0.232 \\
\hline P_B & 0.01049 & 0.0051 & 2.06 & 0.040 \\
\hline LNA & 0.00014 & 0.0005 & 0.28 & 0.782 \\
\hline $\mathrm{CCC}$ & 0.0000005 & 0.0000 & 0.36 & 0.720 \\
\hline OCF_S & $-0.00976(* *)$ & 0.0049 & -1.98 & 0.048 \\
\hline AT & -0.00395 & 0.0053 & -0.75 & 0.453 \\
\hline
\end{tabular}




\begin{tabular}{|r|r|r|r|r|}
\hline & Coef. & Std. Err. & \multicolumn{1}{|c|}{$\mathbf{z}$} & \multicolumn{1}{c|}{ P>z } \\
\hline RB_DIV & $0.00678(* * *)$ & 0.0019 & 3.52 & 0.000 \\
\hline UB_DIV & $-0.00376(* * *)$ & 0.0009 & -4.35 & 0.000 \\
\hline _cons & $0.54440(* * *)$ & 0.0169 & 32.24 & 0.000 \\
\hline
\end{tabular}

Nguồn: Kết quả xử lý từ dữ liệu điều tra

Sau đó nghiên cứu thực hiện các kiểm định cần thiết cho mô hình SEM:

\section{Bảng 5}

Các kiểm định dành cho mô hình SEM

\begin{tabular}{|l|c|}
\hline (i) Kiểm định mô hình tương đương & 0.000 (P - value) \\
\hline (ii) RMSEA & 0.051 \\
\hline (iii) CFI & 0.987 \\
\hline (iv) TLI & 0.950 \\
\hline (v) SRMR & 0.007 \\
\hline (vi) CD & 0.753 \\
\hline
\end{tabular}

Nguồn: Kết quả xử lý từ dữ liệu điều tra

Các chỉ số kiểm định từ (i) tới (vi) là các chỉ số nhằm đánh giá mức độ phù hợp tổng thể của mô hình (được trích xuất từ kiểm định sự phù hợp tổng thể của mô hình: goodness-offit test). Các chỉ số kiểm định từ (i) tới (v) đều đạt yêu cầu đã cho thấy về mặt tổng thể mô hình là phù hợp với dữ liệu thị trường. Ngoài ra, giá trị $\mathrm{CD}$ tương đương với $\mathrm{R}^{2}$ trong mô hình hồi quy bình thường (mục vi) có giá trị 0.753 . Điều này có nghĩa là các biến trong mô hình giải thích được $75.3 \%$ sự biến thiên của của ROE và RISK.

Các kết quả kiểm định được tóm tắt phía trên đã cho thấy mô hình hồi quy đã đảm bảo được các chỉ tiêu kiểm định và có thể sử dụng kết quả hồi quy để thảo luận về tác động của đa dạng hóa kinh doanh tới hiệu quả và rủi ro.

\subsection{Thảo luận kết quả hồi quy}

Căn cứ vào kết quả phân tích hồi quy mô hình SEM, nghiên cứu thảo luận kết quả hồi quy của từng biến, đặc biệt là các biến đại diện cho đa dạng hóa kinh doanh ngành có liên quan và không liên quan như sau:

\subsubsection{Biến số “Đa dạng hóa KD ngành có liên quan” - RB_DIV}

\section{Tác động của RB_DIV tới ROE}

Căn cứ vào kết quả hồi quy theo SEM, hệ số hồi quy của biến RB_DIV mang dấu dương và có ý nghĩa thống kê ở mức $1 \%$ và hệ số tác động là 0.095 . Như vậy, nếu mức độ đa dạng hóa lĩnh vực kinh doanh có liên quan của doanh nghiệp tăng 1 bit thì ROE sẽ tăng $9.5 \%$. Hoặc nếu mức độ đa dạng hóa lĩnh vực kinh doanh có liên quan tăng 0.1 bit thì ROE sẽ tăng $0.95 \%$. Với kết quả hồi quy nêu trên, giả thuyết H1.1 được ủng hộ. Như vậy, có thể các doanh nghiệp trong mẫu nghiên cứu đã tận dụng tốt được các nguồn lực dùng chung để tạo ra được hiệu quả theo quy mô và từ đó có được hiệu quả hoạt động cao hơn. 


\section{Tác động của RB_DIV tới RISK}

Kết quả phân tích thực nghiệm đã ủng hộ giả thuyết H3 khi tác động của RB_DIV tới RISK là dương và mang ý nghĩa thống kê ở mức $1 \%$ với hệ số tác động biên là 0.00678 (= 0.678\%). Có nghĩa, nếu RB_DIV tăng 1 bit mức độ đa dạng hóa thì RISK sẽ tăng xác suất phá sản lên $0.678 \%$; tương ứng, khi RB_DIV tăng 0.1 bit thì RISK tăng $0.0678 \%$. Như vậy, mức tác động biên là không lớn. Từ kết quả tác động của RB_DIV tới ROE và RISK, có thể nhận định rằng việc doanh nghiệp đa dạng hóa sang ngành kinh doanh có liên quan sẽ mang tính đánh đổi giữa hiệu quả và rủi ro phá sản nhưng mức đánh đổi này có vẻ không lớn. Nếu doanh nghiệp tăng 0.1 bit đa dạng hóa thì ROE có cơ hội gia tăng $0.95 \%$ nhưng đánh đổi lại là xác suất phá sản (trong vòng một năm tới) sẽ tăng $0.0678 \%$.

\subsubsection{Biến số "Đa dạng hóa kinh doanh ngành không liên quan" - UB_DIV}

\section{Tác động của UB_DIV tới ROE}

Căn cứ vào kết quả hồi quy theo SEM, hệ số hồi quy của biến UB_DIV mang dấu âm và có ý nghĩa thống kê ở mức $1 \%$ và mức tác động biên bằng 0.123 . Như vậy, nếu mức độ đa dạng hóa kinh doanh ngành không liên quan của doanh nghiệp tăng 1 bit thì ROE sẽ giảm $12.3 \%$. Hoặc nếu mức độ đa dạng hóa lĩnh vực kinh doanh có liên quan tăng 0.1 bit thì ROE sẽ giảm $1.23 \%$.

Với mức độ tác động và chiều tác động tìm thấy trong mô hình ước lượng, có thể thấy việc mở rộng sang các ngành kinh doanh khác quá nhiều so với ngành kinh doanh chính đã khiến doanh nghiệp đối mặt với hai bất lợi: thứ nhất, gặp khó khăn trong việc điều hành cùng lúc các nguồn lực khác nhau, đặc biệt là các nguồn lực mới không giống các nguồn lực cũ (máy móc mới, nhân lực mới, thị trường mới...). Thứ hai, gặp khó khăn trong việc chia sẻ các nguồn lực dùng chung nên khó tiết giảm được chi phí theo quy mô (nghiêm trọng hơn, chi phí có thể sẽ tăng theo quy mô ở giai đoạn đầu của đa dạng hóa ngành không liên quan).

\section{Tác động của UB_DIV tới RISK}

Kết quả ước lượng đã ủng hộ giả thuyết $\mathrm{H} 4$ khi hệ số hồi quy có giá trị -0.00376 và có ý nghĩa thống kê ở mức $1 \%$. Khi đó, nếu mức độ đa dạng hóa ngành không liên quan tăng 1 bit thì rủi ro phá sản giảm $0.376 \%$, hay mức rủi ro này sẽ giảm $0.0376 \%$ nếu mức độ đa dạng hóa tăng 0.1 bit. Mức tác động như vừa nêu là không lớn nhưng phần nào cũng cho thấy việc đa dạng sang ngành kinh doanh khác ngành kinh doanh chính giúp cho doanh nghiệp giảm được rủi ro biến động của chu kỳ kinh doanh. Các ngành kinh doanh khác nhau thường có chu kỳ kinh doanh khác nhau nên rủi ro chu kỳ sẽ giảm và từ đó góp phần làm cho rủi ro phá sản giảm đi.

\section{Kết luận và hàm ý quản trị}

\subsection{Kết luận}

Căn cứ vào cơ sở lý thuyết và các nghiên cứu thực nghiệm trước, nghiên cứu đã xây dựng các giả thuyết nghiên cứu về tác động của hai loại hình đa dạng hóa kinh doanh tới hiệu quả và rủi ro. Và để ước lượng được mối liên hệ trên ở thực nghiệm, nghiên cứu đã sử dụng mô hình ước lượng SEM được điều chỉnh cho dữ liệu bảng cùng với các kiểm định sai phạm mô hình cần thiết. Kết quả ước lượng có một số điểm đáng lưu ý như sau: 
Thứ nhất, mô hình ước lượng SEM trong nghiên cứu đã thỏa mãn hầu hết các kiểm định.

Thứ hai, xét riêng tác động của RB_DIV tới ROE, hệ số hồi quy mang dấu dương và có ý nghĩa thống kê ở mức $1 \%$ và hệ số tác động là 0.095 . Nghiên cứu đã nhận định rằng có thể các doanh nghiệp trong mẫu nghiên cứu đã tận dụng tốt được các nguồn lực dùng chung để tạo ra được hiệu quả theo quy mô và từ đó có được hiệu quả hoạt động cao hơn. Ở góc độ tác động tới RISK, hệ số tác động biên bằng 0.00678 và có ý nghĩa thống kê ở mức $1 \%$ và như vậy doanh nghiệp có doanh thu ở những ngành gần nhau sẽ có chu kỳ kinh doanh dao động khá tương đồng nhau, khi đó rủi ro sẽ tăng cao. Từ kết quả tác động, có thể nhận định rằng việc doanh nghiệp đa dạng hóa sang ngành kinh doanh có liên quan sẽ mang tính đánh đổi giữa hiệu quả và rủi ro phá sản nhưng mức đánh đổi này có vẻ không lớn.

Thứ ba, xét riêng tác động của biến UB_DIV tới ROE, hệ số hồi quy mang dấu âm và có ý nghĩa thống kê ở mức $1 \%$ và mức tác động biên bằng 0.123 . Ở góc độ tác động tới RISK, hệ số hồi quy có giá trị -0.00376 và có ý nghĩa thống kê ở mức $1 \%$. Từ đây, có thể nhận định rằng việc doanh nghiệp đa dạng hóa sang ngành kinh doanh không liên quan cũng sẽ mang tính đánh đổi giữa hiệu quả và rủi ro phá sản nhưng mức đánh đổi này cũng không thực sự đáng kể.

\subsection{Hàm ý quản trị}

Căn cứ vào các kết quả phân tích, nghiên cứu đưa ra một số kiến nghị liên quan tới hoạt động đa dạng hóa kinh doanh của doanh nghiệp như sau:

Thứ nhất, đa dạng hóa kinh doanh, dù ở khía cạnh có liên quan hay không liên quan tới ngành kinh doanh chính đều có tác động nhất định tới hiệu quả và rủi ro phá sản. Nếu doanh nghiệp mở rộng kinh doanh vào các ngành có liên quan tới ngành kinh doanh chính thì $\mathrm{ROE}$ được cải thiện nhưng RISK lại tăng lên. Ngược lại, mở rộng kinh doanh sang các ngành không liên quan sẽ giảm RISK nhưng cũng giảm ROE. Nếu ngành kinh doanh chính của doanh nghiệp đang có rủi ro cao, doanh nghiệp có thể xem xét đa dạng hóa kinh doanh ở các ngành không liên quan để giảm thiểu RISK và chấp nhận đánh đổi lại là ROE sẽ giảm đi. Nếu ngành kinh doanh chính của doanh nghiệp có chiều hướng đi xuống hoặc có mức độ rủi ro ít thì doanh nghiệp nên mở rộng kinh doanh sang các ngành có liên quan.

Thứ hai, xét ở mặt tổng thể, tác động của hai loại hình đa dạng hóa tới ROE và RISK tuy mang tính đánh đổi nhưng việc đánh đổi không mang tính cân xứng. Nếu RB_DIV tăng 0.1 bit thì ROE sẽ tăng $0.95 \%$ và RISK chỉ tăng $0.0678 \%$. Ngược lại, UB_DIV tăng 0.1 bit thì ROE sẽ giảm $1.23 \%$ và RISK chỉ giảm $0.0376 \%$. Có nghĩa, việc gia tăng 0.1 bit RB_DIV có lợi hơn so với việc gia tăng 0.1 bit ở UB_DIV. Hơn nữa, việc gia tăng này không làm gia tăng RISK đáng kể. Như vậy, doanh nghiệp khi đang ở trạng thái hoạt động kinh doanh bình thường thì nên mở rộng sang những ngành kinh doanh có liên quan tới ngành kinh doanh chính.

\section{Tài liệu tham khảo}

Afza, T., \& Nazir, M. S. (2007). Is it better to be aggressive or conservative in managing working capital. Journal of quality and technology management, 3(2), 11-21. 
Alavifar, A., Karimimalayer, M., \& Anuar, M. K. (2012). Structural equation modeling vs multiple regression. Engineering Science and Technology: An International Journal, 2(2), 326-329.

Anderson, J. C., \& Gerbing, D. W. (1988). Structural equation modeling in practice: A review and recommended two-step approach. Psychological Bulletin, 103(3), 411-423.

Andreß, H.-J., Golsch, K., \& Schmidt, A. W. (2013). Applied panel data analysis for economic and social surveys. Berlin, Germany: Springer Science \& Business Media.

Appuhami, B. R. (2008). The impact of firms' capital expenditure on working capital management: An empirical study across industries in Thailand. International Management Review, 4(1), 8-21.

Barney, J. (1991). Firm resources and sustained competitive advantage. Journal of Management, 17(1), 99-120.

BenZion, U., \& Shalit, S. S. (1975). Size, leverage, and dividend record as determinants of equity risk. The Journal of Finance, 30(4), 1015-1026.

Berrington, A., Smith, P., \& Sturgis, P. (2006). An overview of methods for the analysis of panel data. Retrieved October 11, 2017, from http://eprints.ncrm.ac.uk/415/1/MethodsReviewPaperNCRM-007.pdf

Bettis, R. A., \& Hall, W. K. (1982). Diversification strategy, accounting determined risk, and accounting determined return. Academy of Management Journal, 25(2), 254-264.

Blum, M. (1974). Failing company discriminant analysis. Journal of Accounting Research, 12(1), 1-25.

Brush, T. H., Bromiley, P., \& Hendrickx, M. (2000). The free cash flow hypothesis for sales growth and firm performance. Strategic Management Journal, 21(4), 455-472.

Castagna, A., \& Matolcsy, Z. (1978). The relationship between accounting variables and systematic risk and the prediction of systematic risk. Australian Journal of Management, 3(2), 113-126.

Chakravarthy, B. S. (1986). Measuring strategic performance. Strategic Management Journal, $7(5), 437-458$.

Chang, Y., \& Thomas, H. (1989). The impact of diversification strategy on risk-return performance. Strategic Management Journal, 10(3), 271-284.

Cho, H.-J., \& Pucik, V. (2005). Relationship between innovativeness, quality, growth, profitability, and market value. Strategic Management Journal, 26(6), 555-575.

Claessens, S., Djankov, S., \& Nenova, T. (2001). Corporate growth and risk around the world. Financial Crises in Emerging Markets, 305-338.

Connolly, T., Conlon, E. J., \& Deutsch, S. J. (1980). Organizational effectiveness: A multipleconstituency approach. Academy of Management Review, 5(2), 211-218.

Dambolena, I. G., \& Khoury, S. J. (1980). Ratio stability and corporate failure. The Journal of Finance, 35(4), 1017-1026. 
Deakin, E. B. (1972). A discriminant analysis of predictors of business failure. Journal of Accounting Research, 10(1), 167-179.

Deloof, M. (2003). Does working capital management affect profitability of Belgian firms? Journal of Business Finance \& Accounting, 30(34), 573-588.

Dempsey, M. (2010). The book-to-market equity ratio as a proxy for risk: Evidence from Australian markets. Australian Journal of Management, 35(1), 7-21.

Dichev, I. D. (1998). Is the risk of bankruptcy a systematic risk? The Journal of Finance, 53(3), 1131-1147.

Fox, J. (2006). Teacher's corner: Structural equation modeling with the sem package in R. Structural Equation Modeling, 13(3), 465-486.

García-Teruel, P. J., \& Martinez-Solano, P. (2007). Effects of working capital management on SME profitability. International Journal of managerial finance, 3(2), 164-177.

Giacomino, D. E., \& Mielke, D. E. (1993). Cash flows: Another approach to ratio analysis. Journal of Accountancy, 175(3), 55.

Glancey, K. (1998). Determinants of growth and profitability in small entrepreneurial firms. International Journal of Entrepreneurial Behavior \& Research, 4(1), 18-27.

Hall, E. H., \& John, C. H. (1994). A methodological note on diversity measurement. Strategic Management Journal, 15(2), 153-168.

Hennawy, R., \& Morris, R. C. (1983). The significance of base year in developing failure prediction models. Journal of Business Finance \& Accounting, 10(2), 209-223.

Hitt, M. A. (1988). The measuring oforganizational effectiveness: Multiple domains and constituencies. Management International Review, 28-40.

Hoskisson, R. E., \& Hitt, M. A. (1990). Antecedents and performance outcomes of diversification: A review and critique of theoretical perspectives. Journal of Management, 16(2), 461-509.

Jansen, I. P., Ramnath, S., \& Yohn, T. L. (2012). A diagnostic for earnings management using changes in asset turnover and profit margin. Contemporary Accounting Research, 29(1), 221-251.

Jemison, D. B. (1981). The importance of an integrative approach to strategic management research. Academy of Management Review, 6(4), 601-608.

Kaczmarek, J. (2012). The identification and measurement of financial threat vs. the cases of insolvency in the period of Poland's economic transformation. The Business \& Management Review, 2(2), 255.

Kaplan, R. S., \& Cooper, R. (1998). Cost \& effect: Using integrated cost systems to drive profitability and performance. Boston, MA: Harvard Business Press.

Karels, G. V., \& Prakash, A. J. (1987). Multivariate normality and forecasting of business bankruptcy. Journal of Business Finance \& Accounting, 14(4), 573-593. 
Kealhofer, S., \& Bohn, J. R. (1998). Portfolio management of default risk. Net Exposure, 1(2), 12.

Kenny, G. (2009). Diversification strategy: How to grow a business by diversifying successfully. London, UK: Kogan Page Publishers.

Kim, W. C., Hwang, P., \& Burgers, W. P. (1993). Multinationals' diversification and the riskreturn trade-off. Strategic Management Journal, 14(4), 275-286.

Knecht, M. (2013). Diversification, industry dynamism, and economic performance: The impact of dynamic-related diversification on the multi-business firm. Berlin, Germany: Springer Science \& Business Media.

Lang, L. H., \& Stulz, R. M. (1993). Tobin's q, corporate diversification and firm performance. Cambridge, MA: National Bureau of Economic Research.

Lee, B. S., \& Li, M.-Y. L. (2012). Diversification and risk-adjusted performance: A quantile regression approach. Journal of Banking \& Finance, 36(7), 2157-2173.

Lu, Y. (2008). Default forecasting in KMV. Oxford, UK: University of Oxford.

Lubatkin, M., \& Chatterjee, S. (1994). Extending modern portfolio theory into the domain of corporate diversification: does it apply? Academy of Management Journal, 37(1), 109136.

Lubatkin, M., Merchant, H., \& Srinivasan, N. (1993). Construct validity of some unweighted product-count diversification measures. Strategic Management Journal, 14(6), 433-449.

McNeil, A. J., Frey, R., \& Embrechts, P. (2015). Quantitative risk management: Concepts, techniques and tools. Princeton, NJ: Princeton University Press.

McQuown, J. (1997). Market versus accounting-based measures of default risk. Option embedded bonds. Chicago, IL: Irwin Professional Publishing.

McWilliams, A., \& Smart, D. L. (1993). Efficiency v. structure-conduct-performance: Implications for strategy research and practice. Journal of Management, 19(1), 63-78.

Merton, R. C. (1974). On the pricing of corporate debt: The risk structure of interest rates. The Journal of Finance, 29(2), 449-470.

Montgomery, C. A., \& Singh, H. (1984). Diversification strategy and systematic risk. Strategic Management Journal, 5(2), 181-191.

Montgomery, C. A., \& Wernerfelt, B. (1988). Diversification, Ricardian rents, and Tobin's q. The Rand journal of economics, 19(4), 623-632.

Neely, A., Gregory, M., \& Platts, K. (1995). Performance measurement system design: A literature review and research agenda. International Journal of Operations \& Production Management, 15(4), 80-116.

Palich, L. E., Cardinal, L. B., \& Miller, C. C. (2000). Curvilinearity in the diversificationperformance linkage: An examination of over three decades of research. Strategic Management Journal, 21, 155-174. 
Pan, Y., \& David, K. T. (2000). The hierarchical model of market entry modes. Journal of International Business Studies, 31(4), 535-554.

Peirson, G., Brown, R., Easton, S., \& Howard, P. (2014). Business finance. Sydney, Australia: McGraw-Hill Education Australia.

Penrose, E. T. (1959). Theory of the growth of the firm. Oxford, UK: Oxford University Press.

Pils, F. (2009). Diversification, relatedness, and performance. Berlin, Germany: Springer Science \& Business Media.

Ramanujam, V., \& Varadarajan, P. (1989). Research on corporate diversification: A synthesis. Strategic Management Journal, 10(6), 523-551.

Robins, J. A., \& Wiersema, M. F. (2003). The measurement of corporate portfolio strategy: Analysis of the content validity of related diversification indexes. Strategic Management Journal, 24(1), 39-59.

Rumelt, R. P. (1974). Strategy, structure and economic performance. Cambridge, MA: Harvard University Press.

Rumelt, R. P. (1982). Diversification strategy and profitability. Strategic Management Journal, 3(4), 359-369.

Santos, J. B., \& Brito, L. A. L. (2012). Toward a subjective measurement model for firm performance. BAR-Brazilian Administration Review, 9(SPE), 95-117.

Shepherd, W. G. (1986). On the core concepts of industrial economics. In Mainstreams in industrial organization (pp. 23-67). Berlin, Germany: Springer.

Stata, A. (2015). Stata base reference manual release 14. Retrieved October 12, 2017, from http://citeseerx.ist.psu.edu/viewdoc/summary?doi=10.1.1.637.2432

Vasicek, O. A. (1984). Credit valuation. Retrieved October 13, 2017, from http://www.ressourcesactuarielles.net/EXT/ISFA/1226.nsf/0/c181fb77ee99d464c125757a00505078/\$FILE/C redit_Valuation.pdf

Venkatraman, N., \& Prescott, J. E. (1990). The market share-profitability relationship: Testing temporal stability across business cycles. Journal of Management, 16(4), 783-805.

Venkatraman, N., \& Ramanujam, V. (1986). Measurement of business performance in strategy research: A comparison of approaches. Academy of Management Review, 11(4), 801814.

Vishnani, S., \& Shah, B. K. (2007). Impact of working capital management policies on corporate performance - An empirical study. Global Business Review, 8(2), 267-281.

Voelkle, M. C., Oud, J. H., Davidov, E., \& Schmidt, P. (2012). An SEM approach to continuous time modeling of panel data: relating authoritarianism and anomia. Psychological Methods, 17(2), 176-192. 
Wan, W. P., Hoskisson, R. E., Short, J. C., \& Yiu, D. W. (2010). Resource-based theory and corporate diversification: Accomplishments and opportunities. Journal of Management, 37(5), 1335-1368.

Weiss, L. W. (1979). The structure-conduct-performance paradigm and antitrust. University of Pennsylvania Law Review, 127(4), 1104-1140.

Wernerfelt, B. (1984). A resource-based view of the firm. Strategic Management Journal, 5(2), 171-180.

Wooldridge, J. (2015). Introductory econometrics: A modern approach. Toronto, Canada: Nelson Education. 